\title{
CHOQUE CIRCULATÓRIO: DEFINIÇÃO, CLASSIFICAÇÃO, DIAGNÓSTICO E TRATAMENTO
}

\author{
CIRCULATORY SHOCK: DEFINITION, CLASSIFICATION, DIAGNOSIS AND TREATMENT
}

Bruno Ganem Siqueira ${ }^{1} \&$ André Schmidt ${ }^{2}$

\begin{abstract}
1Pós-graduando. ${ }^{2}$ Docente. Divisão de Cardiologia do Departamento de Clínica Médica da Faculdade de Medicina de Ribeirão Preto - USP. Correspondência: Prof. Dr. André Schmidt. Hospital das Clínicas da FMRP-USP. Divisão de Cardiologia Departamento de Clínica Médica. Campus Universitário - USP. CEP: 14048-900 Ribeirão Preto - SP -. Fone: (16) 602-2599 - e-mail: aschmidt@fmrp.usp.br
\end{abstract}

SIQUEIRA BG \& SCHMIDT A. Choque circulátório: Definição, classificação, diagnóstico e tratamento. Medicina, Ribeirão Preto, 36: 145-150, abr./dez. 2003.

RESUMO - Procede sucinta revisão, procurando abordar, de forma sistematizada e prática, a classificação, diagnóstico e tratamento do choque circulatório. São essencialmente enfatizados aspectos conceituais, além de achados clínicos, hemodinâmicos e metabólicos, para melhor caracterização dos estados de choque. Procura-se fundamentar, ainda, princípios básicos da reposição volêmica e intervenções farmacológicas mediante o uso de vasopressores, cardiotônicos e vasodilatadores.

UNITERMOS - Choque. Vasoconstritores. Vasodilatadores. Choque Cardiogênico

\section{1- INTRODUÇÃO}

O choque circulatório faz parte da via final, comum de inúmeras doenças fatais, contribuindo, portanto, para milhões de mortes em todo o mundo.

Trata-se de síndrome clínica, caracterizada pela incapacidade do sistema circulatório em fornecer oxigênio e nutrientes aos tecidos, de forma a atender suas necessidades metabólicas. É o conceito de choque que mostra uma importante relação entre metabolismo e hemodinâmica. Assim, um quadro circulatório, caracterizado por alto débito cardíaco, pode não ser adequado para suprir as necessidades metabólicas numa situação de hipermetabolismo. Por outro lado, um baixo débito cardíaco pode ser adequado para uma situação de hipometabolismo.

Outro aspecto relevante é que nem todos os danos teciduais, resultantes do choque advêm da hipóxia, mas, também, podem decorrer da baixa oferta de nutrientes, reduzida depuração de substâncias tóxicas, maior afluxo de substâncias nocivas aos tecidos, ativação de mecanismos agressores e redução dos mecanismos de defesa.

Para a caracterização e o diagnóstico do estado de choque circulatório, há a necessidade da presença de hipotensão (absoluta ou relativa) e sinais e/ou sintomas de inadequação da perfusão tecidual, explicitados e discutidos adiante.

\section{2- CLASSIFICAÇÃ̃o}

A classificação do choque circulatório obedece a uma finalidade estritamente didática, tendo em vista que a maioria dos quadros de choque mostra mais de um componente em sua evolução (Tabela I):

- Hipovolêmico - caracterizado por baixo volume intravascular, relativo a sua capacitância, ou seja, hipovolemia relativa ou absoluta. $\mathrm{O}$ volume contido no compartimento intravascular é inadequado para a perfusão tecidual. 
- Cardiogênico - existe limitação primária no desempenho cardíaco, decorrente de interferências sobre o inotropismo e/ou cronotropismo. A função de bomba do coração é insuficiente para manter o débito cardíaco em níveis compatíveis com as necessidades metabólicas.

- Obstrutivo - resulta de bloqueio mecânico ao fluxo sangüíneo na circulação pulmonar ou sistêmica.

- Distributivo - caracterizado por inadequação entre a demanda tecidual e a oferta de oxigênio por uma alteração na distribuição do fluxo sangüíneo. Dessa forma, temos tecidos com fluxo sangüíneo elevado em relação à necessidade e outros com fluxo sangüíneo elevado em termos numéricos, mas insuficiente para atender às necessidades metabólicas.

\section{3 - DIAGNÓSTICO}

O diagnóstico de choque circulatório é eminentemente clínico, baseando-se, portanto, numa boa anamnese e exame físico. Para seu diagnóstico, há a necessidade de identificarmos a presença de hipotensão arterial (pressão arterial sistólica (PAS) $<90 \mathrm{mmHg}$, pressão arterial média (PAM) $<60 \mathrm{mmHg}$ ou queda maior que $40 \mathrm{mmHg}$ na PAS), associada a sinais e sintomas de inadequação da perfusão tecidual (Tabela II). A história clínica deve ser direcionada à procura da etiologia, fornecendo, assim, subsídios para a terapêutica mais adequada e eficaz dessa síndrome clínica.

A avaliação laboratorial é essencial para a avaliação da oferta de oxigênio e sua adequação para o metabolismo tecidual. A saturação venosa central, de oxigênio $\left(\mathrm{SvO}_{2}\right)$ retrata a medida do balanço entre a oferta sistêmica e a demanda tecidual de oxigênio, tornando-se muito importante na avaliação e acompanhamento da resposta terapêutica. Os quadros hipodinâmicos aumentam o tempo de trânsito das hemácias na microcirculação, fazendo que a $\mathrm{SvO}_{2}$ torne-se baixa por aumento da extração de $\mathrm{O}_{2}$. Por outro lado, a $\mathrm{SvO}_{2}$ eleva-se em estados com uma captação tecidual deficiente de $\mathrm{O}_{2}$ ou por um quadro hiperdinâmico.

\section{Tabela I - Classificação do choque circulatório}

\section{Hipovolêmico}

- Sangramentos volumosos (politraumatizados, ferimentos com armas de fogo ou arma branca)

- Perda excessiva de líquiidos (diarréia, vômitos, poliúria, queimaduras extensas e febre)

- Seqüiestro de líquiidos em tecidos inflamados (pancreatite, peritonite, colite, pleurite)

- Drenagem de grandes volumes de transudatos (ascite, hidrotórax)

\section{Cardiogênico}

- Miopático (depressão função sistólica)

Infarto agudo do miocárdio

Miocardiopatia dilatada

Depressão miocárdica no choque séptico

\section{- Mecânico}

Regurgitação mitral, aguda

Ruptura do septo interventricular

- Arrítmico

\section{Obstrutivo}

- Tamponamento cardíaco

- Tromboembolismo pulmonar maciço

- Hipertensão pulmonar, importante (primária ou Eisenmenger)

\section{Distributivo}

- Choque séptico

- Choque neurogênico (trauma raquimedular, traumatismo craniano)

- Choque endócrino (hipotireoidismo, hipocortisolismo)

- Anafilaxia (reação de hipersensibilidade a drogas ou contato com substâncias)

- Síndrome vasoplégica, pós-circulação, extracorpórea. 
Tabela II - Manifestações clínicas do choque circulatório (adaptado de B.C.Maciel \& J.A. Marin-Neto. Medicina, Ribeirão Preto, outubro/dezembro 1992) ${ }^{(11)}$

Choque hipodinâmico (frio)

(baixo débito, alta resistência vascular periférica)

- Prostração, ansiedade

- Hipotensão

- Taquicardia

- Pulso filiforme

- Pele: fria, pálida, cianótica

- Sudorese

- Taquipnéia

- Sede, náuseas, vômitos

- Oligúria, anúria

- Inquietude, apreensão, confusão

- Inconsciência (fase tardia)

\section{Choque hiperdinâmico(quente)}

(alto débito, baixa resistência vascular periférica)

- Prostração, ansiedade

- Hipotensão (não acentuada)

- Taquicardia

- Pulso amplo

- Pele: quente, com rubor

- Ausência de sudorese

- Oligúria, diurese moderada

- Febre, calafrios

- Hiperventilação

- Inquietude, apreensão, confusão, coma (raro)

A determinação do lactato sangüíneo constituise em marcador de agressão tecidual secundária à hipóxia ou a diferentes agentes tóxicos. Assim, níveis de lactato normais representam uma oferta de $\mathrm{O}_{2}$, adequada para as necessidades metabólicas, não havendo necessidade, portanto, de metabolismo anaeróbico para a produção de energia. Tal medida ainda não é rotina na maioria dos serviços, mas pode ser de grande valor em formas iniciais, sem grande repercussão clínica e/ou hemodinâmica.

O diagnóstico do tipo de choque circulatório pode ser baseado na determinação de variáveis hemodinâmicas através da monitorização hemodinâmica invasiva, com o uso do cateter de Swan-Ganz, conforme sumarizado na Tabela III.

\section{3- TRATAMENTO}

A sistematização do atendimento inicial é fundamental, de modo que o diagnóstico das anormalidades e seu tratamento sejam feitos passo-a-passo, numa sequiência lógica, fazendo com que as prioridades sejam as mesmas para qualquer tipo de choque.

\section{1- Medidas iniciais}

Dá-se prioridade sempre ao "ABC" onde a letra "A" (do inglês airway) corresponde ao acesso às vias aéreas, de modo a mantê-las pérvias e protegidas contra obstrução por corpos estranhos ou queda da língua, aspiração brônquica de conteúdo gástrico, sangue, fragmentos de dentes, etc.

A letra "B" corresponde à respiração (do inglês breathing), devendo ser mantida a adequada ventilação e oxigenação do paciente. A ventilação mecânica deve ser instituída, quando a ventilação por acessórios não invasivos (cateteres nasais e máscaras específicas) não for suficiente. $\mathrm{O}$ suporte ventilatório mecânico reduz ainda as necessidades metabólicas do paciente, por reduzir o trabalho respiratório, permitindo a redistribuição do fluxo sangüíneo para áreas hipoperfundidas.

Em seguida, dá-se prioridade à letra "C'C" (do inglês, circulation) que corresponde à manutenção da circulação, e deve-se sempre, dar atenção para as causas responsáveis pela instabilidade hemodinâmica, de modo a procurar um tratamento definitivo para o problema.

Tabela III - Variáveis hemodinâmicas e respiratória, nos diversos tipos de choque circulatório. DC = débito cardíaco; $\mathbf{R V P}=$ resistência vascular periférica; $\mathbf{P C P}=$ pressão capilar pulmonar; $\mathrm{PVC}=$ pressão venosa central; $\mathrm{SvO2}$ = saturação venosa central de oxigênio.

\begin{tabular}{|c|c|c|c|c|c|}
\hline Tipo de choque & DC & RVP & PCP & PVC & SvO2 \\
\hline Hipovolêmico & Baixo & Alta & Baixa & Baixa & Baixa \\
\hline Cardiogênico & Baixo & Alta & Alta & Alta & Baixa \\
\hline Obstrutivo & Baixo & Alta & Baixa & Alta & Baixa \\
\hline Distributivo & Alto & Baixa & Alta, normal ou baixa & Alta, normal ou baixa & Alta \\
\hline
\end{tabular}


Juntamente com medidas de restabelecimento e manutenção da circulação (reposição volêmica e agentes vasopressores, vasodilatadores ou inotrópicos) devem ser adotadas medidas definitivas para corrigir o estado de choque, conforme a etiologia encontrada:

1. Cardioversão elétrica para as taquiarritmias.

2. Uso de marcapasso transcutâneo ou transvenoso para as bradiarritmias.

3. Uso de trombolíticos no infarto agudo do miocárdio e tromboembolismo pulmonar.

4. Pericardiocentese ou pericardiotomia no tamponamento cardíaco.

5. Drenagem torácica no pneumotórax hipertensivo.

6. Uso de antibióticos e drenagem cirúrgica de abcesso para o tratamento da infecção no choque séptico.

\section{2- Reposição volêmica}

Independente da etiologia do choque circulatório, a manutenção de pressões de enchimento ventricular, em níveis adequados para produzir um débito cardíaco efetivo, é o objetivo fundamental a ser perseguido. Não apenas a hipovolemia é uma das causas mais comuns de choque, com, também, ela pode existir em determinadas fases de todos os tipos de choque, incluindo o cardiogênico e o distributivo. Conseqüentemente, uma "ressuscitação" volêmica inicial faz-se necessária para expandir o volume intravascular (aumentar o retorno venoso), visando corrigir a hi- potensão, aumentar o volume urinário e melhorar o estado mental; sempre atentar para a deterioração da oxigenação e aparecimento de crepitações pulmonares, que possam sugerir congestão pulmonar (aumento da pressão capilar pulmonar; aumento da pré-carga do ventrículo esquerdo).

O tipo de líquiido utilizado na reposição irá depender da natureza da perda e da intensidade da mesma (Tabela IV). A reposição volêmica pode ser feita com cristalóide (ringer simples, ringer lactato, soro fisiológico) e/ou colóides (sangue e seus derivados, albumina, dextranas e aminos naturais e sintéticos). Os cristalóides apresentam uma capacidade de expansão volêmica bem menor que a dos colóides, já que apenas $1 / 4$ do volume infundido permanece no intravascular. O uso de sangue está reservado para grandes perdas (> 30\% do volume sangüíneo total). Em geral, procura-se manter o hematócrito em $30 \%$ e a taxa de hemoglobina em $10 \mathrm{~g} / \mathrm{dL}$. Pacientes jovens, geralmente, têm condições de suportar taxas menores (até 5 a $7 \mathrm{~g} / \mathrm{dL}$.), seguindo a tendência mundial de evitar expô-los aos riscos das transfusões sangüíneas.

$\mathrm{Na}$ ausência de insuficiência e estenose valvares importantes, embolia pulmonar, aumentos de resistência vascular pulmonar e disfunção ventricular esquerda, a medida da PVC permite estimar a pressão de enchimento do ventrículo direito (VD), constituindo-se numa maneira prática, rápida e econômica de estimar o grau da volemia de paciente, guiar a administração

Tabela IV - Perda estimada de sangue, baseada em parâmetros clínicos, observados na avaliação inicial do paciente com choque hemorrágico (adaptado do manual do curso Advanced Trauma Life Support, organizado pelo Colégio Americano de Cirurgiões).

\begin{tabular}{|l|c|c|c|c|}
\hline & Classe I & Classe II & Classe III & Classe IV \\
\hline Perda Sangüínea (mL) & até 750 & $750-1500$ & $1500-2000$ & $>2000$ \\
\hline Perda sangüínea (\% volume sangüíneo) & até $15 \%$ & $15-30 \%$ & $30-40 \%$ & $>40 \%$ \\
\hline Freqüência cardíaca & $<100$ & $>100$ & $>120$ & $>140$ \\
\hline Pressão arterial & normal & normal & diminuída & diminuída \\
\hline Freqüência respiratória & $14-20$ & $20-30$ & $30-40$ & $>35$ \\
\hline Diurese (mL/h) & $>30$ & $20-30$ & $5-15$ & desprezível \\
\hline Estado mental & ansiedade leve & $\begin{array}{l}\text { ansiedade } \\
\text { moderada }\end{array}$ & ansiedade e confusão & confusão e letargia \\
\hline Reposição volêmica & cristalóide & cristalóide & cristalóide e sangue & cristalóide e sangue \\
\hline
\end{tabular}


de líquiidos e a necessidade ou não do uso de drogas vasoativas. A cateterização da artéria pulmonar, método mais refinado, através do cateter de Swan-Ganz, fornece informações precisas acerca do débito cardíaco (DC), pressão de enchimento do VE e pressão "capilar pulmonar" (PCP), consumo $\left(\mathrm{VO}_{2}\right)$ e oferta (DO2) de oxigênio. Entretanto, é uma técnica bastante onerosa, exigindo local apropriado, equipe bem treinada e equipamento de alto custo, devendo ser reservada como guia para terapêutica, principalmente, em situações de choque circulatório com disfunção ventricular e choque do tipo distributivo, causado por endotoxinas.

Deve-se enfatizar a necessidade de eliminar a hipovolemia e assegurar pressão de enchimento ventricular otimizada, por administração de líqüidos, antes da instituição do tratamento com drogas vasoativas. Isto, porque, nessas condições, a eficácia das mesmas aumenta, a dose necessária diminui e os efeitos adversos tornam-se menos importantes.

\section{3 - Agentes inotrópicos}

\subsection{1- Dobutamina}

Apresenta efeito predominante, betaadrenérgico, responsável por sua ação inotrópica positiva e vasodilatadora periférica, discreta, aumentando o débito cardíaco e diminuindo a resistência vascular periférica. Não libera norepinefrina endógena, induzindo menos taquicardia, arritmias e isquemia miocárdica do que a dopamina e noradrenalina. Não tem efeito vasodilatador, renal, mas o volume urinário e o fluxo renal parecem aumentar igualmente em comparação com a dopamina, sugerindo que o aumento da perfusão renal, secundária ao aumento do débito cardíaco, é o mais importante determinante da manutenção da função renal. A dose usual de dobutamina é de 3 a $20 \mu \mathrm{g} / \mathrm{Kg} / \mathrm{min}$. Nao deve ser usada com pressão sistólica abaixo de $90 \mathrm{mmHg}$, já que pode promover a diminuição da resistência vascular periférica e pressão sistêmica por sua interação com receptores betaadrenérgicos vasculares.

\subsection{2 - Inibidores da fosfodiesterase}

A amrinona e o milrinona são drogas de uso parenteral que têm efeitos hemodinâmicos, semelhantes aos da dobutamina, apresentando ação inotrópica, positiva, por inibição do fosfodiesterase (aumento de AMPc, intracelular). A milirinona (mais comumente usada) é mais potente e possui menor efeito pró-arrítmico que a amrinona. Pode ser usada isolada ou associada com a dobutamina, em paciente com insuficiên- cia cardíaca severa, na dose de ataque de $50 \mu \mathrm{g} / \mathrm{Kg}$ (10 min) e manutenção de 0,375 a $075 \mu \mathrm{g} / \mathrm{Kg} / \mathrm{min}$.

\section{4 - Agentes vasopressores}

\subsection{1 - Noradrenalina}

Mediador adrenérgico natural, com potente efeito constritor venoso e arterial (alfadependente) e modesto efeito inotrópico positivo (beta ${ }_{1}$ dependente). A noradrenalina aumenta, predominantemente, a pressão arterial pela elevação da resistência vascular sistêmica e pode não melhorar, ou até diminuir o débito cardíaco. É utilizada, principalmente, no choque séptico e em condições de choque refratário. Pode ser útil no choque cardiogênico por infarto agudo do miocárdio, porque aumenta pressão na raiz da aorta, melhorando perfusão coronária. Necrose tecidual pode ser observada, se ocorrer extravasamento para o subcutâneo. Dose usual de 0,03 a $2 \mu \mathrm{g} / \mathrm{Kg} / \mathrm{min}$. O uso dessa droga deve ser visto como uma medida temporária e a dose deve ser reduzida ou a administração, descontinuada assim que possível.

\subsection{2 - Dopamina}

Percussor imediato da noradrenalina na via biossintética das catecolaminas. Estimula diretamente receptores alfa e betaadrenérgicos, ao mesmo tempo em que promove liberação de norepinefrina endógena. Doses baixas ( 1 a $3 \mu \mathrm{g} / \mathrm{Kg} / \mathrm{min}$ ) têm efeito basicamente dopaminérgico (aumento do fluxo renal). Doses intermediárias (3 a $10 \mu \mathrm{g} / \mathrm{Kg} / \mathrm{min}$ ) têm efeito, principalmente, beta-estimulante (inotrópico positivo) e doses $>10$ $\mu \mathrm{g} / \mathrm{Kg} / \mathrm{min}$ tem efeito alfa-estimulante (aumento da resistência vascular periférica). Devido a sua maior dificuldade de titulação de dose/efeito desejado, tal amina vasoativa tem sido menos usada. Assim sendo, ao se desejar obter um efeito agonista sobre os receptores adrenérgicos alfa e beta, a melhor opção seria o uso de noradrenalina e dobutamina, respectivamente.

\section{5 - Vasodilatadores}

\subsection{1 - Nitroprussiato}

Vasodilatador balanceado (arterial e venoso), não indutor de taquifilaxia, com rápido início de ação, usado em situações emergenciais, em que se observa aumento da pressão de enchimento do ventrículo esquerdo como insuficiência mitral aguda (disfunção ou ruptura de músculo papilar) ou ruptura de septointerventricular pós-IAM. Só deve ser usado em pacientes com pressão arterial sistólica $>90 \mathrm{mmHg}$. Além 
da hipotensão, pode desencadear taquicardia reflexa, piora da isquemia miocárdica e intoxicação por tiocianato (uso prolongado ou insuficiência renal). A dose deve variar entre 0,25 a $10 \mu \mathrm{g} / \mathrm{Kg} / \mathrm{min}$.

\subsection{2 - Nitroglicerina}

Vasodilatador predominantemente venoso, além de vasodilatador coronário. Extremamente útil em pacientes com insuficiência cardíaca congestiva, que cursam com sinais de congestão pulmonar e princi- palmente, em pacientes com etiologia isquêmica. Inicia-se com $10 \mu \mathrm{g} / \mathrm{min}$, aumentando-se $10 \mu \mathrm{g} / \mathrm{min}$ a cada $5 \mathrm{~min}$ até a dose máxima de $100 \mu \mathrm{g} / \mathrm{min}$.

Em suma, a abordagem diagnóstica e terapêutica do choque circulatório deve levar em conta uma anamnese detalhada, pois os diagnósticos etiológico e do tipo de choque prevalente devem ser feitos de modo a orientar terapêuticas definitivas, que, quando instaladas precocemente, aumentam as chances de reversão do quadro.

SIQUEIRA BG \& SCHMIDTA. Circulatory shock: Definition, classification, diagnosis and treatment. Medicina, Ribeirão Preto, 36: 145-150, apr./dec.2003.

ABSTRACT: This is a brief, systematic and applied review of the classification, diagnosis and treatment of patients with circulatory shock. Conceptual aspects and clinical, hemodynamic and metabolic findings are specially emphasized providing a better shock characterization and understanding. Finally, basic principles of volume expansion and pharmacological interventions (vasopressor and cardiotonic agents, vasodilators) are rationalized.

UNITERMS - Shock. Vasoconstrictor Agents. Vasodilator Agents. Shock, Cardiogenic.

\section{BIBLIOGRAFIA RECOMENDADA}

1 - WHEELER AP \& BERNARD GR. Treating patients with severe sepsis. N Engl J Med 340: 207-214, 1999.

2 - SHOEMAKER WC. Diagnosis and treatment of shock and circulatory dysfunction. In: Textbook of critical care. W.B. Saunders Company, Philadelphia, Chicago, p. 92-114, 1999.

3 - KASINSKI N; ANDREI AM; SCARPINELLA-BUENO MA \& FERNANDES-JUNIOR CJ. Drogas vasoativas. In: KNOBEL $E$, ed. Condutas no paciente grave. Editora Atheneu, São Paulo, p. 111-133, 1999.

4 - KASINSKI N; FERNANDES-JUNIOR CJ \& KNOBEL E. Fisiopatologia do choque. In: KNOBEL E, ed. Condutas no paciente grave. Editora Atheneu, São Paulo, p.3-15, 1999.

5 - KNOBEL E; GONÇALVES-JUNIOR I \& CIRENZA C. Choque cardiogênico. In: KNOBEL E, ed. Condutas no paciente grave. Editora Atheneu, São Paulo, p. 40-56, 1999.

6 - LANDRY DW \& OLIVER JA. The pathogenesis of vasodilatory shock. N Engl J Med 345: 588-595, 2001.

7 - KELLUM AJ \& DECKER JM. Use of dopamine in acute renal faluire: A meta-analysis. Crit Care Med 29: 1526-1530, 2001.

8 - TAYLOR RW. Pulmonary artery catheter Consensus Conference Participants: Pulmonary Artery Catheter Consensus Conference: Consensus Statament. Crit Care Med 25: 910-925, 1997.

9 - HOLLENBERG SM; AHRENS TS; ASTIZ ME; CHALFIN DB; DASTA JF; HEARD SO; MARTIN C; GREGORY SM \& VINCENT GJL. Task Force of the American College of Critical Care Medicine, Society of Critical Care Medicine. Practice parameters for hemodynamic support of sepsis in adult pacients in sepsis. Crit Care Med 27: 639-654, 1999.
10 - KELLUM JA \& PINSKY MR. Use of vasopressor agents in critically ill patients. Cur Opin Crit Care 8: 236-241, 2002.

11 - MACIEL BC \& MARIN-NETO JA. Choque circulatório. Medicina, Ribeirão Preto, 25: 380-388, 1992.

12 - COMITÊ DE TRAUMA: COLÉGIO AMERICANO DE CIRURGIÕES. Choque. In: Suporte avançado de vida no trauma para médicos. $6^{\circ}$ ed. Colégio Americano de Cirurgiões, Chicago, p.87-107, 1997.

13 - SOCIETY OF CRITICAL CARE MEDICINE. ASSOCIAÇÃO DE MEDICINA INTENSIVA BRASILEIRA. Diagnóstico e tratamento do choque: Fundamentos em terapia intensiva. Editora Revinter, São Paulo, p. 51-61, 2000.

14 - MARSON F; PEREIRA GA; PAZIN-FILHO A \& BASILE-FILHO A. A síndrome do choque circulatório. Medicina, Ribeirão Preto, 31: 369-379, 1998.

15 - MENON V \& HOCHMAN JS. Management of cardiogenic shock complicating acute myocardial infarction. Heart 88: 531-537, 2002

16 - VELANOVICH V. Crystalloid versus colloid fluid resuscitation: a meta analysis of mortality. Surgery 105: 65-71, 1990.

17 - COOPER DJ; WALLEY KR; WIGGS RB \& RUSSEL JA. Bicarbonate does not improve hemodynamics in critically ill patients who have lactic acidosis. Ann Intern Med 112: 492-498, 1990 